\title{
Integration of a Proximity Detection Prototype into a VO Developed with PANGEA
}

\author{
Carolina Zato, Alejandro Sánchez, Gabriel Villarrubia, Javier Bajo, \\ and Sara Rodríguez \\ Departamento Informática y Automática, Universidad de Salamanca, Salamanca, Spain \\ \{carol_zato, asanchezyu,gvg, jbajope, srg\}@usal.es
}

\begin{abstract}
This article presents a proximity detection prototype that uses ZigBee technology developed using the agent's platform PANGEA (Platform for Automatic coNstruction of orGanizations of intElligent Agents). PANGEA is an agent platform to develop open multiagent systems, specifically those including organizational aspects such as virtual agent organizations. The platform allows the complete management of organizations and offers tools to the end user. Due to the specific characteristics of this prototype, PANGEA is the perfect candidate to develop the prototype that will be included in the future in an integral system primarily oriented to facilitate the integration of people with disabilities into the workplace.
\end{abstract}

Keywords: Zigbee, proximity detection, virtual organizations, agent platform, multiagent system.

\section{Introduction}

Within the field of technologies specifically developed to facilitate the lives of people with disabilities, there have been many recent advances that have notably improved their ability to perform daily and work-related tasks, regardless of the type and severity of the disability. This article presents a proximity detection prototype, specifically developed for a work environment, which can facilitate tasks such as activating and personalizing the work environment; these apparently simple tasks are in reality extremely complicated for some people with disabilities.

This prototype has been developed using PANGEA. There are many different platforms available for creating multiagent systems that facilitate the work with agents [10][11][12][13]; however our aim is to have a tool that allows to create an increasingly open and dynamic multiagent system (MAS). This involves adding new capabilities such as adaption, reorganization, learning, coordination, etc. Virtual Organizations (VOs) of agents [8] [9] emerged in response to this idea; they include a set of agents with roles and norms that determine their behavior, and represent a place where these new capabilities will assume a critical role. Possible organizational topologies and aspects such as communication and 
coordination mechanisms determine in large part the flexibility, openness and dynamic nature that a multiagent system can offer. All these features are taken into account with the new platform PANGEA, for this reason the prototype has been integrated into a MAS developed with the mentioned platform.

The rest of the paper is structured as follows: The next section introduces the PANGEA platform used in the development of this prototype. Section 3 presents the most important characteristics of the prototype. Section 4 explains the case study and finally, in section 5 some conclusions are presented.

\section{PANGEA Overview}

PANGEA is a service oriented platform that allows the MAS to create with it to take the maximum advantage of the distribution of resources. To this end, all services are implemented as Web Services. This makes it possible for the platform to include both a service provider agent and a consumer agent, thus emulating a client-server architecture. The provider agent knows how to contact the web service; once the client agent's request has been received, the provider agent extracts the required parameters and establishes the contact. Once received, the results are sent to the client agent.

This leads us to one of the most important features that characterize the platform; PANGEA allows to create and integrate any kind of agent, does not matter the language, the functionality or other characteristics. The own agents of the platform are implemented with Java, nevertheless the agents of the detection prototype are implemented in .NET and nesC.

Using PANGEA, the platform will automatically launch the following agents:

- OrganizationManager: the agent is responsible for the actual management of organizations and suborganizations. It is responsible for verifying the entry and exit of agents, and for assigning roles. To carry out these tasks, it works with the OrganizationAgent, which is a specialized version of this agent.

- InformationAgent: the agent is responsible for accessing the database containing all pertinent system information.

- ServiceAgent: the agent is responsible for recording and controlling the operation of services offered by the agents.

- NormAgent: the agent that ensures compliance with all the refined norms in the organization.

- CommunicationAgent: the agent is responsible for controlling communication among agents, and for recording the interaction between agents and organizations.

- $\quad$ Sniffer: manages the message history and filters information by controlling communication initiated by queries.

Initially, the platform creates a general VO but thanks to the organizational concepts related to this model, it is possible to create suborganizations. This is the reason why the OrganizationAgents are included, its function is to help the 
OrganizationManager to support all the suborganizations. Each suborganization or work unit is automatically provided with an OrganizationAgent by the platform during the creation of the suborganization. This OrganizationAgent is similar to the OrganizationManager, but is only responsible for controlling the suborganizationn, and can communicate with the OrganizationManager if needed. If another suborganization is created hierarchically within the previous suborganization, it will include a separate OrganizationAgent that communicates with the OrganizationAgent from the parent organization. These agents are distributed hierarchically in order to free the OrganizationManager of tasks. This allows each OrganizationAgent to be responsible for a suborganization although, to a certain extent, the OrganizationManager can always access information from all of the organizations. Each agent belongs to one suborganization and can only communicate with the OrganizationAgent from its own organization; this makes it possible to include large suborganizational structures without overloading the AgentManager. All of the OrganizationAgents from the same level can communicate with each other, unless a specific rule is created to prevent this.

Following this modeling way, the detection proximity prototype is included as a suborganization called DetectionProximityOrganization with his own OrganizacionAgent. In the next section, all the architecture will be explained.

\section{Integration of the Detection Proximity Prototype}

ZigBee sensors are used to deploy the detection prototype. ZigBee is a low cost, low power consumption, two-way wireless communication standard that was developed by the ZigBee Alliance [5]. It is based on the IEEE 802.15.4 protocol [2], and operates on the ISM (Industrial, Scientific and Medical) band at $868 / 915 \mathrm{MHz}$ and a $2.4 \mathrm{GHz}$ spectrum. Due to this frequency of operation among devices, it is possible to transfer materials used in residential or office buildings while only minimally affecting system performance [1]. Although this system can operate at the same frequency as Wi-Fi devices, the possibility that it will be affected by their presence is practically null, even in very noise environments (electromagnetic interference). ZigBee is designed to be embedded in consumer electronics, home and building automation, industrial controls, PC peripherals, medical sensor applications, toys and games, and is intended for home, building and industrial automation purposes, addressing the needs of monitoring, control and sensory network applications [5]. ZigBee allows star, tree or mesh topologies. Devices can be configured to act as network coordinator (control all devices), router/repeater (send/receive/resend data to/from coordinator or end devices), and end device (send/receive data to/from coordinator) [6]. One of the main advantages of this system is that, as opposed to GPS type systems, it is capable of functioning both inside and out with the same infrastructure, which can quickly and easily adapt to practically any applied environment. 


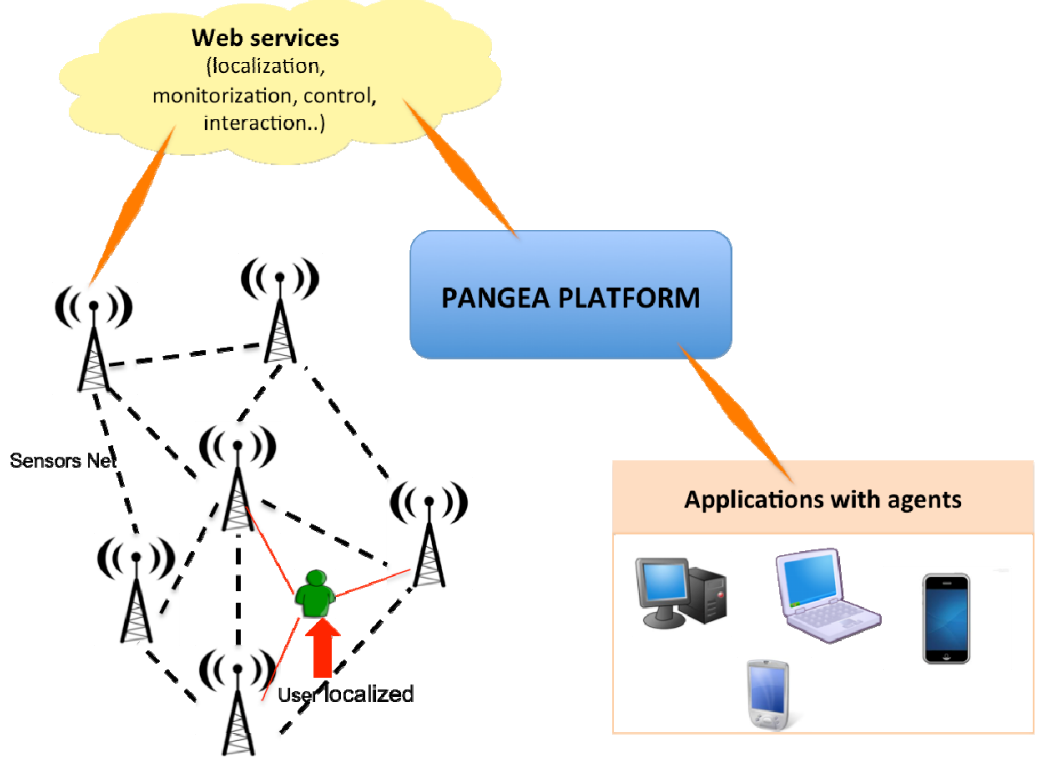

Fig. 1 Diagram of the Zigbee network

\subsection{Architecture}

The agents involved in the specialized suborganization developed inside PANGEA are:

- ZigbeeManagerAgent: it manages communication and events and is deployed in the server machine.

- ProfileManagerAgent: it is responsible for managing user profiles and is also deployed in the server machine.

- ClientComputerAgent: these are user agents located in the client computer and are responsible for detecting the user's presence with ZigBee technology, and for sending the user's identification to the ZigbeeManagerAgent. These agents are responsible for requesting the profile role adapted for the user to the ProfileManagerAgent.

- DatabaseAgent: the detection proximity system uses a database, which stores data related to the users, sensors, computer equipment and status, and user profiles. It can also communicate with the InformationAgent of PANGEA.

- ZigBeeCoordinatorAgent: it is an agent included in a ZigBee device responsible for coordinating the other ZigBee devices in the office. It is connected to the server by a serial port, and receives signals from each of the ZigBee tags in the system. 


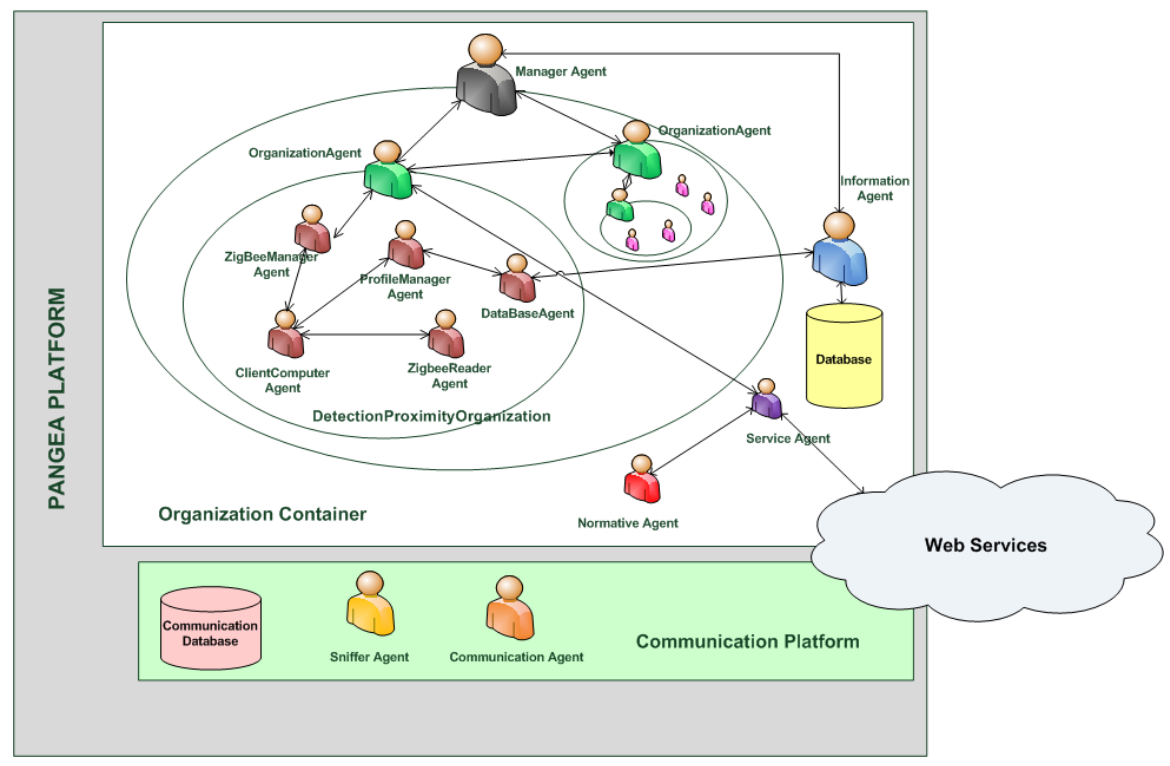

Fig. 2 System architecture

- ZigBeeReaderAgent: these agents are included in several ZigBee devices that are used to detect the presence of a user. Each ZigBeeReaderAgent is located in a piece of office equipment (computer).

Every user in the proposed system carries a Zigbee tag, which is detected by a ZigBeeReaderAgent located in each system terminal and continuously in communication with the ClientComputerAgent. Thus, when a user tag is sufficiently close to a specific terminal (within a range defined according to the strength of the signal), the ZigBeeReaderAgent can detect the user tag and immediately send a message to the ClientComputerAgent, which is coordinated by the ZigBeeCoordinatorAgent.

The system uses a LAN infrastructure that uses the wake-on-LAN protocol for the remote switching on and off of equipment. Wake-on-LAN/WAN is a technology that allows a computer to be turned on remotely by a software call. It can be implemented in both local area networks (LAN) and wide area networks (WAN) [4]. It has many uses, including turning on a Web/FTP server, remotely accessing files stored on a machine, telecommuting, and in this case, turning on a computer even when the user's computer is turned off [7].

\section{Case Study}

This paper presents a proximity detection system that it is used by people with disabilities to facilitate their integration in the workplace. The main goal of the system is to detect the proximity of a person to a computer using ZigBee 
technology. This allows an individual to be identified, and for different actions to be performed on the computer, thus facilitating workplace integration: automatic switch on/off of the computer, identifying user profile, launching applications, and adapting the job to the specific needs of the user. Thanks to the Zigbee technology the prototype is notably superior to existing technologies using Bluetooth, infrareds or radiofrequencies, and is highly efficient with regards to detection and distance. Additionally, different types of situations in a work environment were taken into account, including nearby computers, shared computers, etc.

In our Case Study we have a distribution of computers and laptops in a real office environment, separated by a distance of 2 meters. The activation zone is approximately $90 \mathrm{~cm}$, a distance considered close enough to be able to initiate the activation process. It should be noted that there is a "Sensitive Area" in which it is unknown exactly which computer should be switched on; this is because two computers in close proximity may impede the system's efficiency from switching on the desired computer. Tests demonstrate that the optimal distance separating two computers should be at least $40 \mathrm{~cm}$.

The proposed proximity detection system is based on the detection of presence by a localized sensor called the control point (where the ZigBeeReaderAgent is deployed), which has a permanent and known location. Once the Zigbee tag carried by the person has been detected and identified, its location is delimited within the proximity of the sensor that identified it. Consequently, the location is based on criteria of presence and proximity, according to the precision of the system and the number of control points displayed.

The parameter used to carry out the detection of proximity is the RSSI (Received Signal Strength Indication) [14], a parameter that indicates the strength of the received signal. This force is normally indicated in $\mathrm{mW}$ or using logarithmic units $(\mathrm{dBm}) .0 \mathrm{dBm}$ is equivalent to $1 \mathrm{~mW}$. Positive values indicate a signal strength greater than $1 \mathrm{~mW}$, while negative values indicate a signal strength less than $1 \mathrm{~mW}$.

Under normal conditions, the distance between transmitter and receiver is inversely proportional to the RSSI value measured in the receiver; in other words, the greater the distance, the lower the signal strength received. This is the most commonly used parameter among RTLS [15].

RSSI levels provide an appropriate parameter for allowing our system to function properly. However, variations in both the signal transmission and the environment require us to define an efficient algorithm that will allow us to carry out our proposal. This algorithm is based on the use of a steps or measurement levels (5 levels were used), so that when the user enters the range or proximity indicated by a RSSI level of -50 , the levels are activated. While the values received are less than the given range, each measurement of the system activates a level. However, if the values received fall outside the range, the level is deactivated. When the maximum number of levels has been activated, the system interprets this to mean that the user is within the proximity distance of detection and wants to use the computer equipment. Consequently, the mechanisms are activated to remotely switch on both the computer and the profile specific to the user's disability. 
The system is composed of 5 levels. The tags default to level 0 . When a user moves close to a marker, the level increases by one unit. The perceptible zone in the range of proximity gives an approximate RSSI value of -50 . If the user moves away from the proximity area, the RSSI value is less than -50 , resulting in a reduction in the level. When a greater level if reached, it is possible to conclude that the user has remained close to the marker, and the computer will be turned on.

On the other hand, reaching an initial level of 0 means that the user has moved a significant distance away from the workspace, and the computer is turned off.

\section{Conclusions}

This prototype is specifically oriented to facilitate the integration of people with disabilities into the workplace. The detection and identification of a user makes it possible to detect any special needs, and for the computer to be automatically adapted for its use. This allows the system to define and manage the different profiles of people with disabilities, facilitating their job assimilation by automatically switching on or off the computer upon detecting the user's presence, or initiating a procedure that automatically adapts the computer to the personal needs of the user.

Thanks of the PANGEA platform, the prototype could be designed and deployed easily since the platform itself provides agents and tools for the control and management of any kind of open MAS or VO. Moreover, the platform allows to deploy different agents, indeed those included into the mobile devices and performs the communication with the agents embedded into the Zigbee sensors.

The prototype is part of complete and global project in which different tools for helping disabled people will be included. Using the platform PANGEA, that models all the services as Web Services and promotes scalability, the addition in the future of all those services that conform the global project will be easier.

Acknowledgements. This project has been supported by the Spanish CDTI. Proyecto de Cooperación Interempresas. IDI-20110344.

\section{References}

1. Huang, Y., Pang, A.: Comprehensive Study of Low-power Operation in IEEE 802.15.4. In: Proceeding of the 10th ACM Symposium on Modeling, Analysis and Simulation of Wireless and Mobile Systems, Chaina, Crete Island, Greece (2007)

2. Singh, C.K., et al.: Performance evaluation of an IEEE 802.15.4 Sensor Network with a Star Topology (2008)

3. Universidad Pontificia de Salamanca [En línea] (2011), http: / /www . youtube. com/watch?v=9iYX-xney6E

4. Lieberman, P.: Wake on LAN Technology, White paper (2011), http: / /www. liebsoft.com/pdfs/Wake_On_LAN.pdf

5. ZigBee Standards Organization: ZigBee Specification Document 053474r13. ZigBee Alliance (2006) 
6. Tapia, D.I., De Paz, Y., Bajo, J.: Ambient Intelligence Based Architecture for Automated Dynamic Environments. In: Borrajo, D., Castillo, L., Corchado, J.M. (eds.) CAEPIA 2007, vol. 2, pp. 151-180 (2011)

7. Nedevschi, S., Chandrashekar, J., Liu, J., Nordman, B., Ratnasamy, S., Taft, N.: Skilled in the art of being idle: reducing energy waste in networked systems. In: Proceedings of the 6th USENIX Symposium on Networked Systems Design and Implementation, Boston, Massachusetts, April 22-24, pp. 381-394 (2009)

8. Ferber, J., Gutknecht, O., Michel, F.: From Agents to Organizations: An Organizational View of Multi-agent Systems. In: Giorgini, P., Müller, J.P., Odell, J.J. (eds.) AOSE 2003. LNCS, vol. 2935, pp. 214-230. Springer, Heidelberg (2004)

9. Foster, I., Kesselman, C., Tuecke, S.: The anatomy of the grid: Enabling scalable virtual organizations. Int. J. High Perform. Comput. Appl. 15(3), 200-222 (2001)

10. Agent Oriented Software Pty. Ltd. JACK ${ }^{\mathrm{TM}}$ Intelligent Agents Teams Manual. Agent Oriented Software Pty. Ltd. (2005)

11. Hübner, J.F.J.: Moise+ Programming organisational agents with Moise+ \& Jason. In: Technical Fora Group at EUMAS 2007

12. Giret, A., Julián, V., Rebollo, M., Argente, E., Carrascosa, C., Botti, V.: An open architecture for service-oriented virtual organizations. In: Braubach, L., Briot, J.-P., Thangarajah, J. (eds.) ProMAS 2009. LNCS, vol. 5919, pp. 118-132. Springer, Heidelberg (2010)

13. Galland, S.: JANUS: Another Yet General-Purpose Multiagent Platform. In: Seventh AOSE Technical Forum, Paris (2010)

14. Zhao, J., Zhang, Y., Ye, M.: Research on the Received Signal Strength Indication Location Algorithm for RFID System. In: International Symposium on Communications and Information Technologies, ISCIT 2006, pp. 881-885 (2006)

15. Park, D.-J., Choi, Y.-B., Nam, K.-C.: RFID-Based RTLS for Improvement of Operation System in Container Terminals. In: Asia-Pacific Conference on Communications, APCC 2006, pp. 1-5 (2006) 